PROCEEDINGS OF THE

AMERICAN MATHEMATICAL SOCIETY

Volume 131, Number 4, Pages 1297-1302

S 0002-9939(02)06633-9

Article electronically published on November 6, 2002

\title{
CHARACTERIZATIONS OF PARACOMPACTNESS AND LINDELÖFNESS BY THE SEPARATION PROPERTY
}

\author{
YUKINOBU YAJIMA
}

(Communicated by Alan Dow)

\begin{abstract}
The separation property in our title is that, for two spaces $X$ and $Y$, any two disjoint closed copies of $X$ in $Y$ are separated by open sets in $Y$. It is proved that a Tychonoff space $X$ is paracompact if and only if this separation property holds for the space $X$ and every Tychonoff space $Y$ which is a perfect image of $X \times \beta X$ (where $\beta X$ denotes the Stone-Cech compactification of $X$ ). Moreover, we give a characterization of Lindelöfness in a similar way under the assumption of paracompactness.
\end{abstract}

\section{INTRODUCTION}

Throughout this paper, all spaces are assumed to be Hausdorff. Let $Y$ be a space. We say that two disjoint subsets $A$ and $B$ in $Y$ are separated by open sets in $Y$ if there are two disjoint open sets $U$ and $V$ in $Y$ such that $A \subset U$ and $B \subset V$. Here we deal with the separation property that, for two spaces $X$ and $Y$, any two disjoint closed copies of $X$ in $Y$ are separated by open sets in $Y$.

Arhangel'skiı $\mathrm{Ar}$ gave a survey on relative topological properties and relative topological spaces. Since this subject includes not only some applications but also many problems, it has been studied in many papers. As an application of it, Arhangel'skiı and Tartir [AT] characterized compactness by the relative regularity property. Moreover, they raised two problems which suggested a characterization of Lindelöfness by the separation property above (see [AT, Problems 1 and 2]). Bella and Yaschenko [BY] answered these problems as follows. Matveev, Pavlov and Tartir [MPT] also independently proved almost the same result.

Theorem 1.1 ([BY], $\overline{\mathrm{MPT}}]$ ). For a Tychonoff space $X$, the following are equivalent.

(a) $X$ is Lindelöf.

(b) For every Tychonoff space $Y$, any two disjoint closed copies of $X$ in $Y$ are separated by open sets in $Y$.

The survey $\mathrm{Ar}$ mainly dealt with relative normality: For a space $Y$, we say that a subspace $X$ in $Y$ is (strongly) normal in $Y$ if for any disjoint closed sets $A$ and $B$ in $Y$ (in $X$ ), $A \cap X$ and $B \cap X$ are separated in $Y$. Their proofs of Theorem 1.1

Received by the editors May 30, 2000 and, in revised form, November 12, 2001.

2000 Mathematics Subject Classification. Primary 54B10, 54D20.

Key words and phrases. Paracompact, separated by disjoint open sets, completely separated, Lindelöf. 
simultaneously yield an answer to [Ar Problems 49 and 50] (= $\mathrm{AT}$, Problems 3 and 4]) for this property, which is

Theorem 1.2 ([BY], [MPT]). For a normal space $X$, the following are equivalent.

(a) $X$ is Lindelöf or almost compact.

(b) $X$ is (strongly) normal in every larger Tychonoff space $Y$.

In Theorems 1.1 and 1.2, the space $Y$ ranges over the class of all Tychonoff spaces containing $X$. In fact, the spaces $Y$ constructed in [BY], [MPT] are so complicated that they cannot be represented in terms of only the space $X$. So it seems to be natural to consider the following question:

Question. Can the class over which $Y$ ranges in Theorems 1.1 or 1.2 be restricted to some smaller class $\mathcal{C}(X)$ of spaces related to $X$ ?

On the other hand, the author [Ya] proved that a Tychonoff space $X$ is Lindelöf if and only if the subspace $(X \times \alpha X) \cup(\alpha X \times X)$ of the square $(\alpha X)^{2}$ is normal for some compactification $\alpha X$ of $X$. This result is based on Tamano's Theorem for paracompactness in [T1], T2] stated below. These two results give us some suggestions about how to find the class $\mathcal{C}(X)$. The purpose of this paper is to give an answer to our Question for Theorem 1.1, which will be divided into two steps.

In the first step, we prove that paracompactness can be characterized in a similar way as in Theorem 1.1. In the characterization, $\mathcal{C}(X)$ is given as the class consisting of all Tychonoff spaces that are perfect images of the product $X \times \beta X$ of the space $X$ and its Stone-Čech compactification $\beta X$. This result itself might be more interesting than the original purpose.

In the second step, by the first one, we may assume the paracompactness of $X$ for our purpose. Under this assumption, we can also characterize Lindelöfness in a similar way as Theorem 1.1, where $\mathcal{C}(X)$ is given by the class consisting of the subspaces $(X \times \gamma X) \cup(\gamma X \times X)$ of $(\gamma X)^{2}$ for all compactifications $\gamma X$ of $X$.

Thus, our results do not only give an extension of Theorem 1.1, but also our proof seems to be rather simpler than those in [BY], [MPT].

\section{PARACOMPACTNESS}

Lemma 2.1. Let $X$ be a space and $C$ a compact space. Let $F$ be a closed subspace in $X \times C$. Let $F_{x}$ be a non-empty subset of $C$ such that $\{x\} \times F_{x}=F \cap(\{x\} \times C)$ for each $x \in X$. Let $Y$ be the quotient space of $X \times C$ by identifying $\{x\} \times F_{x}$ with a point $\left\langle x, p_{x}\right\rangle$ for each $x \in X$. Let $q: X \times C \rightarrow Y$ be the quotient map. Then we have

(1) $q$ is a perfect map;

(2) $\left\{\left\langle x, p_{x}\right\rangle: x \in X\right\}$ is a closed subspace of $Y$ which is homeomorphic to $X$;

(3) if $X$ is Tychonoff, then $Y$ is also Tychonoff.

Proof. (1): Let $\pi_{X}: X \times C \rightarrow X$ be the projection. Let $M$ be a closed subset in $X \times C$. Note that $q^{-1} q(M)=M \cup\left(\left[\pi_{X}(M \cap F) \times C\right] \cap F\right)$. Since $\pi_{X}$ is a closed map, $q^{-1} q(M)$ is a closed set in $X \times C$. Since $q$ is a quotient map, it follows that $q$ is a closed map. It is obvious that $q^{-1}(y)$ is compact for each $y \in Y$. Hence $q$ is a perfect map.

(2): Let $P=\left\{\left\langle x, p_{x}\right\rangle: x \in X\right\}$. Since each $F_{x}$ is non-empty, we have $P=q(F)$. Since $q$ is a closed map, $P$ is a closed subset in $Y$. Consider the natural map $\varphi: P \rightarrow X$ defined by $\varphi\left(x, p_{x}\right)=x$ for each $x \in X$. Then it is clear that $\varphi$ 
is a continuous bijection. Note that $\varphi(A)=\pi_{X}\left(q^{-1}(A)\right)$ for each $A \subset P$. Let $R$ be a closed subset in $P$. Since $R$ is closed in $Y$ and $\pi_{X}$ is a closed map, it follows that $\varphi(R)$ is closed in $X$. Hence $\varphi$ is a closed map, which means that it is a homeomorphism.

(3): Let $y \in Y$ and let $K$ be a non-empty closed set in $Y$ with $y \notin K$. In case $y \neq\left\langle x, p_{x}\right\rangle$ for each $x \in X$, we can easily find a continuous function $f: Y \rightarrow[0,1]$ such that $f(y)=0$ and $f(K)=\{1\}$. Hence, we only need to consider the case that $y=\left\langle x, p_{x}\right\rangle$ for some $x \in X$. Let $L=q^{-1}(K)$. By $q^{-1}(y)=F \cap(\{x\} \times C)$, we have $F \cap L \cap(\{x\} \times C)=\emptyset$. Since $C$ is compact, there are an open neighborhood $U$ of $x$ in $X$ and a binary open cover $\{V, W\}$ of $C$ such that

$$
(U \times V) \cap L=\emptyset \text { and }(U \times W) \cap F=\emptyset .
$$

Let $A=\pi_{C}(F \cap(U \times C))$ and $B=\pi_{C}(L \cap(U \times C))$, where $\pi_{C}: X \times C \rightarrow C$ is the projection. Since $V \cap B=\emptyset$ and $W \cap A=\emptyset$, it follows that $\mathrm{Cl}_{C} A \cap \mathrm{Cl}_{C} B=\emptyset$. Since $C$ is normal, there is a continuous function $\psi: C \rightarrow[0,1]$ such that $\psi(A)=\{0\}$ and $\psi(B) \subset\{1\}$. On the other hand, since $X$ is Tychonoff, there is a continuous function $\varphi: X \rightarrow[0,1]$ such that $\varphi(x)=0$ and $\varphi(X \backslash U) \subset\{1\}$. Now, define a function $g: X \times C \rightarrow[0,1]$ by $g(s, t)=\min \{1, \varphi(s)+\psi(t)\}$ for each $\langle s, t\rangle \in X \times C$. Then $g$ is continuous. By the definition of $g$, we have

$$
g\left(q^{-1}(y)\right) \subset g(\{x\} \times A)=\{0\} \quad \text { and } \quad g\left(q^{-1}(K)\right)=\{1\} .
$$

Since $g$ is constant on $q^{-1}(z)$ for each $z \in Y$, we can define a function $h: Y \rightarrow[0,1]$ such that $g=h \circ q$. Since $g$ is continuous and $q$ is a quotient map, $h$ must be continuous. Now, it is easily verified that $h(y)=0$ and $h(K)=\{1\}$. Hence the space $Y$ is Tychonoff.

Two disjoint subsets $A$ and $B$ in a space $X$ are completely separated if there is a continuous function $f: X \rightarrow[0,1]$ such that $f(A) \subset\{0\}$ and $f(B) \subset\{1\}$. For a Tychonoff space $X$, we denote by $\beta X$ the Stone-Cech compactification of $X$, and by $\Delta_{X}$ the diagonal of $X$, that is, $\Delta_{X}=\{\langle x, x\rangle: x \in X\}$.

Let us restate Tamano's Theorem, which plays important roles here.

Tamano's Theorem ([T1], [T2]). For a Tychonoff space $X$, the following are equivalent.

(a) $X$ is paracompact.

(b) $X \times \beta X$ is normal.

(c) For each compact subset $K$ of $\beta X-X, X \times K$ and $\Delta_{X}$ are completely separated in $X \times \beta X$.

Burke $[\mathrm{Bu}]$ gave another proof of Tamano's Theorem in his own way getting the following result. We also use his idea to prove our Theorem 2.3 below.

Lemma $2.2([\mathrm{Bu}])$. A space $X$ is paracompact if and only if every open cover $\mathcal{U}$ of $X$ has an open refinement $\mathcal{V}$ such that for each $x \in X$ there are an open neighborhood $W_{x}$ of $x$ and a finite subcollection $\mathcal{W}$ of $\mathcal{U}$ with $\operatorname{St}\left(W_{x}, \mathcal{V}\right) \subset \cup \mathcal{W}$ and $x \in \bigcap \mathcal{W}$.

Theorem 2.3. For a Tychonoff space $X$, the following are equivalent.

(a) $X$ is paracompact.

(b) For every compactification $\gamma X$ of $X$, any two disjoint closed copies of $X$ in $X \times \gamma X$ are completely separated in $X \times \gamma X$. 
(c) For every Tychonoff space $Y$ which is a perfect image of $X \times \beta X$, any two disjoint closed copies of $X$ in $Y$ are separated by open sets in $Y$.

Proof. (a) $\Rightarrow$ (b) and (a) $\Rightarrow$ (c): These are obvious.

(b) $\Rightarrow$ (a): Let $K$ be any compact subset of $\beta X-X$. Then the quotient space of $\beta X$ by identifying $K$ with a point $p$ is a compactification of $X$, which is denoted by $\gamma X$. Since $p \in \gamma X-X$, it follows that $X \times\{p\}$ and $\Delta_{X}$ are disjoint closed copies of $X$ in $X \times \gamma X$. So $X \times\{p\}$ and $\Delta_{X}$ are completely separated in $X \times \gamma X$. Hence $X \times K$ and $\Delta_{X}$ are completely separated in $X \times \beta X$. It follows from Tamano's Theorem that $X$ is paracompact.

(c) $\Rightarrow$ (a): For each open set $U$ in $X$, let $U^{*}$ be an open set in $\beta X$ with $U^{*} \cap$ $X=U$. Let $\mathcal{U}$ be an open cover of $X$. We may assume that $\mathcal{U}$ has no finite subcover. Let $F=X \times \beta X-\bigcup\left\{U \times U^{*}: U \in \mathcal{U}\right\}$. Let $F_{x} \subset \beta X$ be such that $\{x\} \times F_{x}=F \cap(\{x\} \times \beta X)$ for each $x \in X$. Then each $F_{x}$ is non-empty. Let $Y$ be the quotient space of $X \times \beta X$ by identifying $\{x\} \times F_{x}$ with a point $\left\langle x, p_{x}\right\rangle$ for each $x \in X$.

By Lemma 2.1 (1) and (3), $Y$ is a Tychonoff space and a perfect image of $X \times \beta X$. Let $P=\left\{\left\langle x, p_{x}\right\rangle: x \in X\right\}$. It follows from Lemma $2.1(2)$ that $P$ is a closed subset of $Y$ which is homeomorphic to $X$. It is easily checked that $\Delta_{X}$ is a closed subset in $Y$ which is homeomorphic to $X$. Hence $P$ and $\Delta_{X}$ are disjoint closed copies of $X$ in $Y$. By the assumption, there is an open set $G$ in $Y$ such that $\Delta_{X} \subset G \subset \mathrm{Cl}_{Y} G \subset Y-P$. Without loss of generality, we may assume that $G=\bigcup\left\{V_{\alpha} \times V_{\alpha}^{*}: \alpha \in \Lambda\right\}$, where $V_{\alpha}$ is an open set in $X$ for each $\alpha \in \Lambda$. Now, we put $\mathcal{U}^{*}=\left\{U^{*}: U \in \mathcal{U}\right\}, \mathcal{V}=\left\{V_{\alpha}: \alpha \in \Lambda\right\}$ and $\mathcal{V}^{*}=\left\{V_{\alpha}^{*}: \alpha \in \Lambda\right\}$. Pick any point $x \in X$, and fix it.

Claim. $p_{x} \notin \mathrm{Cl}_{Y_{x}} \operatorname{St}\left(U_{x}^{*}, \mathcal{V}^{*}\right)$ for some open neighborhood $U_{x}$ of $x$ in $X$, where $Y_{x}$ denotes the quotient space of $\beta X$ by identifying $F_{x}$ with a point $p_{x}$.

Proof. Since $\left\langle x, p_{x}\right\rangle \notin \mathrm{Cl}_{Y} G$, we can take an open neighborhood $H$ of $\left\langle x, p_{x}\right\rangle$ in $Y$ with $H \cap G=\emptyset$. There are an open neighborhood $U_{x}$ of $x$ in $X$ and an open set $W_{x}$ in $\beta X$ such that $\{x\} \times F_{x} \subset U_{x} \times W_{x} \subset q^{-1}(H)$. Then we have that $\left(U_{x} \times W_{x}\right) \cap\left(V_{\alpha} \times V_{\alpha}^{*}\right)=\emptyset$ for each $\alpha \in \Lambda$. Since $X$ is dense in $\beta X, U_{x}^{*} \cap V_{\alpha}^{*} \neq \emptyset$ implies $W_{x} \cap V_{\alpha}^{*}=\emptyset$ for each $\alpha \in \Lambda$. Hence we have $W_{x} \cap \operatorname{St}\left(U_{x}^{*}, \mathcal{V}^{*}\right)=\emptyset$. This implies that $p_{x} \notin \mathrm{Cl}_{Y_{x}} \operatorname{St}\left(U_{x}^{*}, \mathcal{V}^{*}\right)$.

Note that $\beta X-F_{x}=\operatorname{St}\left(x, \mathcal{U}^{*}\right)$. By the Claim, the compact set $\mathrm{Cl}_{Y_{x}} \operatorname{St}\left(U_{x}^{*}, \mathcal{V}^{*}\right)$ is contained in $Y_{x}-\left\{p_{x}\right\}=\operatorname{St}\left(x, \mathcal{U}^{*}\right)$. There is a finite subcollection $\mathcal{S}$ of $\mathcal{U}^{*}$ such that $\operatorname{St}\left(U_{x}^{*}, \mathcal{V}^{*}\right) \subset \bigcup \mathcal{S}$ and $x \in \cap \mathcal{S}$. Here, let $\mathcal{W}=\{S \cap X: S \in \mathcal{S}\}$. Then $\mathcal{W}$ is a finite subcollection of $\mathcal{U}$ such that $x \in \cap \mathcal{W}$. Moreover, we have $\operatorname{St}\left(U_{x}, \mathcal{V}\right)=\operatorname{St}\left(U_{x}^{*}, \mathcal{V}^{*}\right) \cap X \subset \bigcup \mathcal{S} \cap X=\bigcup \mathcal{W}$. Hence it follows from Lemma 2.2 that $X$ is paracompact.

\section{LINDELÖFNESS}

A space $X$ is $\omega_{1}$-compact (or the extent of $X$ is countable) if every closed discrete subset in $X$ is at most countable. According to [AT], [MPT], Gordienko [Go proved that if a Tychonoff space $X$ is normal in every larger Tychonoff space, then $X$ is $\omega_{1}$-compact.

Here, for a Tychonoff space $X$ and a compactification $\gamma X$ of $X$, let us regard $(X \times \gamma X) \cup(\gamma X \times X)$ as the subspace of the square $(\gamma X)^{2}$. Modifying the proof of [Ya, Lemma 2.2], we obtain the following. 
Lemma 3.1. If for every compactification $\gamma X$ of a Tychonoff space $X$, any two disjoint closed copies of $X$ in $(X \times \gamma X) \cup(\gamma X \times X)$ are separated by open sets in it, then $X$ is $\omega_{1}$-compact.

Proof. Assume that $X$ is not $\omega_{1}$-compact. There is an uncountable closed discrete subset $D$ in $X$. Let $D^{*}$ be the set of all accumulation points of $D$ in $\beta X$. Consider the quotient space of $\beta X$ by identifying $D^{*}$ with a point $p$. As this is a compactification of $X$, we denote it by $\gamma X$. Note that $p \in \gamma X-X$ and that $\gamma X$ contains the one-point compactification $A(D)=D \cup\{p\}$ of $D$ as a closed subspace. Let $Z=(X \times \gamma X) \cup(\gamma X \times X)$ and let $Y=A(D)^{2}-\{\langle p, p\rangle\}$. Then $Y$ is a closed subspace of $Z$. Since $X \times\{p\}$ and $\{p\} \times X$ are disjoint closed subsets in $Z$, there are disjoint open sets $U$ and $V$ in $Z$ such that $X \times\{p\} \subset U$ and $\{p\} \times X \subset V$. Hence $D \times\{p\}$ and $\{p\} \times D$ are separated by open sets $U \cap Y$ and $V \cap Y$ in $Y$. On the other hand, it is easily seen that $D \times\{p\}$ and $\{p\} \times D$ cannot be separated by open sets in $Y$ (for example, see the proof of [Gr, Lemma 2.5]).

Theorem 3.2. For a Tychonoff space $X$, the following are equivalent.

(a) $X$ is Lindelöf.

(b) For every compactification $\gamma X$ of $X$, any two disjoint closed copies of $X$ in $(X \times \gamma X) \cup(\gamma X \times X)$ are completely separated in it.

(c) $X$ is paracompact, and for every compactification $\gamma X$ of $X$, any two disjoint closed copies of $X$ in $(X \times \gamma X) \cup(\gamma X \times X)$ are separated by open sets in it.

Proof. (a) $\Rightarrow$ (b): Since $(X \times \gamma X) \cup(\gamma X \times X)$ is Lindelöf, it is normal.

(b) $\Rightarrow$ (c): It suffices to show that $X$ is paracompact, which can be verified in the same way as in the proof of Theorem 2.3. Let $K$ be any compact subset of $\beta X-X$. We let $\gamma X$ be the compactification of $X$ by identifying $K$ with a point $p$ in $\beta X$. Then $X \times\{p\}$ and $\Delta_{X}$ are completely separated in $(X \times \gamma X) \cup(\gamma X \times X)$. Thus, they are also completely separated in $X \times \gamma X$. Hence $X \times K$ and $\Delta_{X}$ are completely separated in $X \times \beta X$. It follows from Tamano's Theorem that $X$ is paracompact.

(c) $\Rightarrow$ (a): It follows from Lemma 3.1 that $X$ is $\omega_{1}$-compact. Thus, we have only to recall that every paracompact and $\omega_{1}$-compact space is Lindelöf.

As a consequence, Theorem 1.1 in the Introduction immediately follows from our Theorems 2.3 an 3.2 .

\section{ACKNOWLEDGEMENT}

The author would like to thank Haruto Ohta for his help in preparing this paper.

\section{REFERENCES}

[Ar] A. V. Arhangel'skiü, Relative topological properties and relative topological spaces, Topology and Appl. 70 (1996), 87-99. MR 97f:5403

[AT] A. V. Arhangel'skiĭ and J. Tartir, A characterization of compactness by a relative separation property, Q and A in General Topology. 14 (1996), 49-52. MR 96m:54037

[Bu] D. K. Burke, Covering properties, Handbook of Set-theoretic Topology (K. Kunen and J. E. Vaughan, eds), North-Holland, Amsterdam, 1984, pp. 347-422. MR 86e:54030

[BY] A. Bella and I. V. Yaschenko, Lindelöf property and absolute embeddings, Proc. Amer. Math. Soc. 127 (1999), 907-913. MR 99f:54031

[Go] I. J. Gordienko, A characterization of the Lindelöf property by relative paracompactness, Spaces, Mappings and Functions, MGU, Moscow, 1992, pp. 40-44. 
[Gr] G. Gruenhage, Covering properties on $X^{2}-\Delta$, W-sets, and compact subsets of $\Sigma$ products, Topology and Appl. 17 (1984), 287-304. MR 86e:54029

[MPT] M. V. Matveev, O. I. Pavlov and J. K. Tartir, On relative normal spaces, relatively regular spaces, and on relative property (a), Topology and Appl. 93 (1999), 121-129. MR 2000b:54020

[T1] H. Tamano, On paracompactness, Pacific J. Math. 10 (1960), 1043-1047. MR 23:A2186

[T2] H. Tamano, On compactifications, J. Math. Kyoto Univ. 1 (1962), 161-193. MR 25:5489

[Ya] Y. Yajima, Analogous results to two classical characterizations of covering properties by products, Topology and Appl. 84 (1998), 3-7. MR 98m:54027

Department of Mathematics, Kanagawa University, Yokohama 221-8686, Japan

E-mail address: yajimy01@kanagawa-u.ac.jp 